\title{
Spanish validation and factor structure of the Birth Satisfaction Scale-Revised (BSS-R)
}

Borja Romero-Gonzalez ${ }^{\mathrm{a}, \mathrm{b}}$, Maria Isabel Peralta-Ramirez ${ }^{\mathrm{a}, \mathrm{b}^{*}}$, Rafael A. Caparros-Gonzalez ${ }^{\mathrm{c}}$, Almudena Cambil-Ledesma ${ }^{\mathrm{d}}$, Caroline J. Hollins Martin ${ }^{\mathrm{e}}$, Colin R. Martin ${ }^{\mathrm{f}}$

Affiliations: aBrain, Mind and Behavior Research Center (CIMCYC), Faculty of Psychology, University of Granada, Granada, Spain.

${ }^{b}$ Department of Personality, Assessment and Psychological Treatment, University of Granada, Granada, Spain.

'Nursing Department, University of Jaen, Jaen, Spain.

${ }^{\mathrm{d}}$ Delivery Area, Obstetrics and Gynecology Service, Virgen de Las Nieves Hospital, Granada, Spain.

eSchool of Health and Social Care, Edinburgh Napier University (ENU), Sighthill Campus, Edinburgh EH11 4BN, Scotland, UK.

fFaculty of Health Sciences, University of Hull, HU6 7RX, United Kingdom.

${ }^{*}$ Corresponding author: Maria Isabel Peralta-Ramirez. Department of Personality, Assessment and Psychological Treatment, University of Granada, Granada, Spain. Email: mperalta@ugr.es Conflict of Interest: None declared.

Ethical Approval: Ethical approval for the study was granted by the Ethic Committee of Biomedical Research of Andalusian Government, according to the World Medical Association Declaration of Helsinki.

Funding Sources: This work was supported by the by the I+D Project "PSI2015-63494-P” of the Spanish Ministry of Science and Innovation, co-supported by funds/European Regional Development Fund (ERDF) - a way to build Europe. Besides, Mr. Borja Romero-Gonzalez has been awarded with an individual research grant (Spanish Ministry of Economy, Industry and Competitiveness, FPI Program, reference number BES-2016-077619)

Acknowledgements: Thank you to every pregnant woman who joined and completed our study. This study is a part of a Doctoral Thesis of Mr. Borja Romero-Gonzalez. 


\section{Spanish validation and factor structure of the Birth Satisfaction Scale-Revised}

(BSS-R)

Objective: To translate and validate a Spanish-language version of the Birth

Satisfaction Scale-Revised (BSS-R) and describe key measurement properties.

Design: A cross-sectional instrument validation design examining factor structure, validity and reliability.

Setting: Three public hospital sites in Spain.

Participants: 202 women who had given birth within the past four weeks provided complete questionnaire data for analysis.

Measurements and findings: Measures included the Spanish version of the BSS-R (SBSS-R) and the Perceived Stress Scale (PSS). The tri-dimensional measurement model of the BSS-R was found to offer a good fit to Spanish data. Known-groups discriminant validity was found to be excellent with women experiencing a non-intervention delivery having higher S-BSS-R scores ( $\mathrm{p}<0.05)$ compared to those having an intervention. Women who had no pain control were found to have greater S-BSS-R total and S-BSSR women's attributes and stress experienced sub-scale scores compared to those who received pain control $(\mathrm{p}<0.05)$. Total scale internal reliability was also found to be acceptable. Evidence for good divergent and convergent validity was also found across total and sub-scale S-BSS-R scores.

Key conclusions: The S-BSS-R was found to have generally good to excellent psychometric properties and represents a valid and reliable translation of the original version of the BSS-R for use in Spanish-speaking populations. The development of the S-BSS-R enables comparison of birth satisfaction with international study data where the BSS-R is used. 
Implications for research: The relationship of both delivery type and pain control to birth satisfaction represent important areas for further research. 


\section{Introduction}

Childbirth represents an emotionally complex experience that not only represents interrelated physiological and psychological processes, but also the unique experience itself is circumscribed and influenced by the quality of health care received (Bell and Andersson, 2016). Women's experience of labour can have a significant impact on their own lives, and wider social relationships, specifically their partner, previous children, and of course the new born infant (Conde et al., 2008; Ford and Ayers, 2009).

Birth satisfaction represents a multidimensional construct, which unsurprisingly, is influenced by such diverse phenomena as discrepancy between childbirth expectations and reality of childbirth (Hollins Martin and Fleming, 2011); the quality of health care, including emotional support, communication and doctor-patient relationship received (Bryanton et al., 2008); active participation in decision-making about labour, control perception and stress experienced during labour and birth (Goodman et al., 2004).

Satisfaction with medical processes and health systems has grown as contemporary area of interest, particularly in terms of the relationship to actual quality of care (Fowler and Patterson, 2013). Unsurprisingly, appropriate use of available health services improves when the perception and satisfaction with the service is positive (Mpembeni et al., 2007; Srivastava et al., 2015). Satisfaction with healthcare represents such an important aspect of globalisation, that assessment is central to service evaluation, measurement usually being conducted using validated self-completion questionnaires due to their high reliability and low cost (Blazquez et al., 2017; Konerding, 2016; Marin-Morales et al., 2013). Several questionnaires have been developed as an assessment tool for measuring women’s birth satisfaction (Hollins Martin and Fleming, 2011). 
The Birth Satisfaction Scale-Revised (BSS-R) was designed from an extensive psychometric assessment (Hollins Martin and Martin, 2014) of items derived from a theoretically-informed and thematically-derived long-form version, the Birth Satisfaction Scale (BSS;(Hollins Martin and Fleming, 2011). The BSS-R comprises 10 items and consists of 3 subscales: (1) quality of care provided, (2) personal attributes of women and (3) stress experienced during childbirth.

The growing awareness of the importance of satisfaction with childbirth reflected in clinical outcomes, measured using short, valid, and reliable and theoretically supported measure such as the BSS-R has facilitated international interest, application and endorsement of this questionnaire (Martin et al., 2017; The International Consortium for Health Outcome Measurement, 2016). Thus, the BSS-R has been validated and translated into several versions including Greek, Australian, American and Turkish (Barbosa-Leiker et al., 2015; Goncu Serhatlioglu et al., 2018; Jefford et al., 2018; Martin et al., 2017; Vardavaki et al., 2015).

Due to the importance and usefulness of the BSS-R and because of the lack of short assessment tools available to measure birth satisfaction within a Spanish population, the purpose of the current investigation was to develop and validate a Spanish version of the BSS-R (S-BSS-R), examining key psychometric parameters of reliability and validity. Specifically, our objectives were to:

1. Demonstrate the replicability of the tri-dimensional measurement model of the BSS-R to the S-BSS-R.

2. Evaluate the divergent validity of the S-BSS-R

3. Evaluate the convergent validity of the S-BSS-R.

4. Evaluate the known-groups discriminant validity of the S-BSS-R. 
5. Investigate the potential relationship between the S-BSS-R total and sub-scale scores and pain control during labour.

6. Evaluate the internal consistency of the Quality of Care (QC), Women's Attributes (WA), and Stress Experienced during Child-bearing (SE) sub-scales for the S-BSS-R.

\section{Method}

\section{Design}

A cross-sectional survey design utilising purposive sampling. Participants were informed of the study when they gave birth. Those who agreed to participate read and signed the informed consent form. Those who consented but chose not to complete the study questionnaires at the same time took the questionnaires and returned them within one month of delivery. Participants were recruited from XXXX Hospital (XXX), XXX Hospital (XXX) and XXX Hospital (XXX).

\section{Ethical approval}

Ethical approval for the study was granted by the Ethic Committee of Biomedical Research of the XXX.

\section{Measures}

The BSS-R (Hollins Martin and Martin, 2014), comprises ten items scored on a fivepoint Likert type scale with possible responses being: strongly agree, agree, neither agree or disagree, disagree, strongly disagree. A number of items are reverse-scored, with higher scores on the BSS-R total scale and sub-scales indicating comparatively greater birth satisfaction. The items relate to three sub-scales: stress experienced during child-bearing (SE; 4 items); quality of care (QC; 4 items); and women's attributes (WA; 
2 items) and combined produce an overall total BSS-R score. The psychometric profile of the BSS-R has been found to be valid and reliable across different versions (BarbosaLeiker et al., 2015; Burduli et al., 2017; Goncu Serhatlioglu et al., 2018; Jefford et al., 2018; Martin et al., 2017; Martin et al., 2016; Vardavaki et al., 2015).

The Perceived Stress Scale (PSS; Cohen et al., 1983; Remor, 2006) is a fourteen item scale designed to assess the perception of stress during the previous month. Each item scores on a 5 -point Likert scale $(0=$ never, $1=$ almost never, 2 = once in a while, $3=$ often, 4 = very often). Interpretation consists of adding up each item, higher scores indicative of comparatively greater perceived stress. The Cronbach’s alpha reliability coefficient of the Spanish version is $\alpha=0.8$.

\section{Development of the Spanish version of the BSS-R: Translation process}

The translation of BSS-R into Spanish was done by the backtranslation method. In the first step, the UK English-language version of the BSS-R (Hollins Martin and Martin, 2014) was translated into Spanish by two researchers with a proficient level in English. During the second step, another researcher with proficient levels in both English and Spanish and who was unconnected to the research, translated the scale into English. A pilot study $(\mathrm{N}=10)$ was carried out to obtain feedback about comprehension and interpretation of items prior to agreement of the final version. A draft version of the SBSS-R were administered to a total of 10 women who gave birth in the previous four weeks. Then, they were asked if every item was clear, understandable and concise, and if the full questionnaire was easy to understand and complete. The full sample of 10 women reported not having encountered any difficulties while completing the S-BSS-R and neither were any of the S-BSS-R items indicated to be ambiguous or requiring clarification of meaning. This version of the S-BSS-R was then used as the final version of the S-BSS-R for psychometric evaluation. 


\section{Statistical analysis}

\section{Confirmatory factor analysis}

Consistent with previous investigations (Barbosa-Leiker et al., 2015; Burduli et al., 2017; Goncu Serhatlioglu et al., 2018; Hollins Martin and Martin, 2014; Jefford et al., 2018; Martin et al., 2017; Vardavaki et al., 2015), Objective 1 was addressed using Confirmatory Factor Analysis (CFA; Brown, 2015) to evaluate the tri-dimensional measurement model (Hollins Martin and Martin, 2014) of the BSS-R. Maximumlikelihood estimation was used to evaluate the CFA model (Brown, 2015; Kline, 2011). The comparative fit index (CFI; Bentler, 1990), the root mean squared error of approximation (RMSEA; Steiger and Lind, 1980), the square root mean residual (SRMR; Hu and Bentler, 1999) are the most widely used model fit indices in CFA and were thus selected for the current study.

\section{Divergent validity}

Divergent validity was evaluated by correlating S-BSS-R sub-scale scores with participant age. No statistically significant correlation between S-BSS-R sub-scale scores and this parameter is predicted.

\section{Convergent validity}

Convergent validity was evaluated by examination of correlations between the S-BSS-R total score and sub-scale scores and the PSS. It was predicted that S-BSS-R total and SE scales would be significantly correlated with the PSS. It was also predicted that the QC sub-scale would be significantly correlated with the PSS, but that the comparative strength of the association would be less than the S-BSS-R total and SE sub-scale score association. Finally, no statistically significant relationship was predicted between the 
WA sub-scale and the PSS (thus an additional evaluation of divergent validity for this sub-scale).

\section{Known-groups discriminant validity}

Consistent with previous BSS-R validation studies (Jefford et al., 2018; Vardavaki et al., 2015) known-groups discriminant validity was evaluated by comparison of BSS-R sub-scale and total scores as a function of delivery type, (i.) unassisted vaginal delivery, vs. (ii.) assisted delivery (instrument, Caesarean section). Statistically significant higher S-BSS-R total, SE and WA sub-scale scores are predicted in women having an unassisted vaginal delivery (UVD). Given that the original BSS-R development study and the Australian validation study demonstrated no significant differences between groups as a function of delivery type on the QC sub-scale, in contrast to a large US study and a Greek translation/validation study, where UVD was associated with significantly higher QC sub-scale scores, no specific prediction is made in terms of directionality for this specific S-BSS-R sub-scale.

\section{Pain control}

Known-group discriminant validity was further evaluated by stratifying groups on the basis of whether pain control (epidural/nitrous oxide/massage) was received or no pain control given. It was predicted that a statistically significant difference in S-BSS-R total and sub-scale scores would be observed on the basis of pain control status. The directionality of predicted difference is not apriori specified.

\section{Internal consistency}

Cronbach’s coefficient alpha (Cronbach, 1951) was used to evaluate the internal consistency of S-BSS-R sub-scales and the total scale with a threshold of 0.70 or greater indicating acceptability (Kline, 2000). 


\section{Results}

\section{Participants}

Two-hundred and eight native Spanish-speaking women who had given birth within the past four weeks consented to take part in the study. Six participants were excluded due to being multivariate outliers on BSS-R item scores as determined by Mahalanobis distances greater than threshold $\left(\chi^{2}>29.59\right)$. Multivariate normal S-BSS-R data was thus available for analysis for $\mathrm{N}=202$ participants. Participant mean age was 32.86 (SD 4.75), range = 19-47 years. Pregnancy duration was 39.57 (SD 1.51) weeks and just over half of participants had had their first baby $(\mathrm{N}=104, \%=51 \%)$. The mean time of completion of self-report measures was 7 days (SD 8.88) postpartum. S-BSS-R subscale and total scores are summarised in Table 1. Comparison with the original UK BSS-R total and sub-scale mean scores using the one-sample $t$-test revealed no statistically significant differences.

\section{TABLE 1}

Descriptive characteristics of each S-BSS-R item are summarised in Table 2 and reveal no evidence of excessive skew or kurtosis based on Kline’s (2011) thresholds of 3 (skew) and 10 (kurtosis).

\section{TABLE 2}

All correlations between the S-BSS-R total score and SE, WA, and QE sub-scales were highly statistically significant, respectively, $r=0.91, p<0.001, r=0.78, p<0.001$ and $r$ $=0.64, p<0.001$. Correlations between SE sub-scale and the WA $(r=0.61, p<0.001)$ 
and QC ( $r=0.39, p<0.001)$ sub-scales were highly statistically significant, as was the correlation between WA and QC sub-scales $(r=0.29, p<0.001)$. When compared with the correlations reported in the original UK BSS-R development study (Hollins Martin and Martin, 2014) using the method of Diedenhofen and Musch (2015), no statistically significant differences between S-BSS-R and UK BSS-R total or sub-scale correlations were observed, with the exception of S-BSS-R total score and SE which was significantly higher in the current study (Table 3).

\section{TABLE 3}

\section{Confirmatory factor analysis}

The three-factor measurement model of the BSS-R was observed to offer a good fit to the data $\left(\chi_{(\mathrm{df}=32)}^{2}=62.55, p<0.05, \mathrm{CFI}=0.94, \mathrm{RMSEA}=0.07, \mathrm{SRMR}=0.06\right)$.

\section{Divergent validity}

No statistically significant correlations between S-BSS-R total and sub-scale scores and participant age, (S-BSS-R total score $r=-0.06, p=0.36$, SE $r=-0.06, p=0.43$, WA $r=$ 0.04, $p=0.54$, QE $r=-0.05, p=0.46$ ) were observed.

\section{Convergent validity}

Statistically significant positive correlations were observed between S-BSS-R total, SE and QC sub-scores, and the PSS, $(r=-0.20, p=0.006, r=-0.20, p=0.006$ and $r=0.14, p=$ 0.05 respectively). No statistically significant correlation was observed between the WA sub-scale and the PSS ( $r=0.11, p=0.13)$. 


\section{Known-groups discriminant validity}

Statistically significant differences were observed on all S-BSS-R sub-scales and the total score with women having an unassisted vaginal delivery having higher S-BSS-R scores compared to those that experienced an intervention (Table 4).

\section{TABLE 4}

Women who received no pain control were observed to have significantly higher SBSS-R sub-scale and total scores compared to those who received pain control (Table 5).

TABLE 5

\section{Internal consistency}

Total scale, SE, WA and QC sub-scale Cronbach's alphas are summarised in Table 6. Comparison with Cronbach alpha reported by Hollins Martin and Martin (2014) revealed the alpha of the QA sub-scale to be significantly lower than that of the original UK version with no other statistically significant differences observed between studies.

\section{TABLE 6}




\section{Discussion}

The aim of this study was to validate a Spanish-language version of the BSS-R (Hollins Martin and Martin, 2014) in a Spanish sample of women. Findings from this investigation are generally consistent with the original UK version.

CFA demonstrated good fit to the three-factor measurement model of the original version. These observations are also consistent with other translated non-English language version which show good fit to the BSS-R tri-dimensional measure model (Goncu Serhatlioglu et al., 2018; Vardavaki et al., 2015), thus offering further compelling evidence of the transferability of the birth satisfaction conceptual model of the BSS-R to another non-English language and thus conferring confidence in the factor structure of the Spanish version.

Also observed was no statistically significant differences between S-BSS-R total and sub-scale mean scores and those reported by Hollins Martin and Martin (2014) thus revealing a further layer of consistency between Spanish and UK versions, a finding of particular interest given the ICHOM (The International Consortium for Health Outcome Measurement, 2016) recommended use of the BSS-R within the Pregnancy and Childbirth standard set in allowing robust and valid comparisons between countries, associated health economies and health outcomes. Similar findings were observed for the comparison of S-BSS-R correlations between sub-scales/total score and those reported by Hollins Martin and Martin (2014), with the exception of correlations between the S-BSS-R stress sub-scale and the S-BSS-R total score (0.91) which were significantly higher than those reported in the original BSS-R development study (0.86). However, it should be noted that, in terms of common variance explained, this difference is modest (83\% vs. 74\%) and viewed within the context of no other 
statistically significant differences in sub-scale correlations being observed between studies, would again suggest good evidence of equivalence to the original measure.

Adopting the same known-groups discriminant validity testing paradigm of previous BSS-R validation studies, it is noted that our findings are also consistent with previous investigations. Women who had an unassisted vaginal delivery were observed to have significantly higher S-BSS-R total and sub-scale scores compared to those who had an assisted/intervention delivery. Unpacking these observations in comparison to previous BSS-R studies, provides some useful comparative insights into the distinct facets of birth satisfaction as measured by the BSS-R. The finding of the S-BSS-R total score being significantly higher in the unassisted group would appear to be a universal finding in published BSS-R studies (Fleming et al., 2016; Hollins Martin and Martin, 2014; Jefford et al., 2018) and additional pooled data reported in a meta-analysis of BSS-R studies (Martin and Hollins Martin, 2018). This finding in isolation, highlights both the need and desirability of women to be adequately and appropriately informed about birth choices and options in relation to delivery type, particularly in relation to health economies that foster a culture of interventionism and medicalisation of the birth experience. We note that, in one large US BSS-R study in which women elected to have their babies in birth centres, essentially counter to the dominant federal interventionist/medical culture of the US, that the largest effect size for comparisons for unassisted vs. assisted delivery were observed in favour of unassisted delivery. Similarly, in relation to the S-BSS-R SE sub-scale women experiencing an unassisted delivery scored significantly higher compared to those receiving an intervention, thus, and again consistent with other studies (Fleming et al., 2016; Hollins Martin and Martin, 2014; Jefford et al., 2018), indicating that assisted delivery is associated with less satisfaction and more stress. Given the relationship of stress as a fundamental 
component to distinct perinatal mental health presentations, ranging from postpartum posttraumatic stress disorder (Ali, 2018; Dikmen-Yildiz et al., 2017; Shlomi Polachek et al., 2016), tokophobia (Goutaudier et al., 2018; Striebich et al., 2018) and postnatal depression (Dennis et al., 2018; Pampaka et al., 2018), this finding yields valuable data regarding the potential implications of delivery type on psychological well-being and the development of psychiatric disturbance. Focusing upon the S-BSS-R QC sub-scale we noted that women who had an assisted delivery were significantly less satisfied in terms of quality of care received compared to those who had an unassisted delivery. Reflecting on these findings in relation to previous BSS-R studies again yield some valuable insights that may be of relevance when evaluating and redesigning the system of care provision within Spain. It is noted that in the UK (Hollins Martin and Martin, 2014) and Australian (Jefford et al., 2018) BSS-R studies no significant differences were observed on the BSS-R QC sub-scale as a function of delivery type. This contrasts with the large US study (Fleming et al., 2016) and the Greek study (Vardavaki et al., 2015) where statistically significant differences were observed on the BSS-R subscale in favour of unassisted delivery. The most logical explanation for differences between studies on this single index of birth satisfaction is likely to be the intrinsic characteristics of the healthcare system itself. Therefore, within the context of Spain, the findings are illuminating in relation to actual and real differences that women may encounter as a function of non-intervention/intervention that are individually evaluated in relation to the quality of care received. Comparison with other studies internationally using the same index of birth satisfaction and instrument measurement framework is thus incredibly useful in terms of clarifying the potential differential impact of specific elements of the care package as they influence the women's perceptions and experiences of care. 
Extending the established known-groups discriminant validity testing paradigm associated with the BSS-R to novel group comparisons, this is the first validation study to examine group differences in terms of pain control. Statistically significant differences were observed as a function of pain control status on S-BSS-R total and SE and WA sub-scales. On these birth satisfaction measures women experienced greater satisfaction in the no pain control group compared to those receiving pain control, and in the case of the S-BSS-R total and SE sub-scale scores, these differences were highly statistically significant. Pain control within the context of birth represents a complex topic in terms of not only physiological elements but also psychological aspects that may be anticipated to play a role in pain experienced (Christiaens et al., 2010; Guszkowska, 2014). A perspective that a positive view of pain and accepting it as a part of the process of childbirth could increase birth satisfaction may be a potential explanation (Whitburn et al., 2014), though as highlighted the topic is complex, the debate rich and dissonances on the topic and possible mechanisms involved common (Van der Gucht and Lewis, 2015).

A surprising finding given the generally acceptable internal consistency of the QC subscale in other BSS-R validation studies (Hollins Martin and Martin, 2014; Jefford et al., 2018) was the sub-optimal Cronbach’s alpha observed in the current study.

Interestingly, one other validation study of the BSS-R (Vardavaki et al., 2015), found a similar alpha level for the QC sub-scale. Given that alpha is influenced by the number of items within the scale, this, with other mediating factors, for example cultural context, might play a role in the lower than anticipated alpha observation of this subscale. It is of note that in the Turkish version of the BSS-R (Goncu Serhatlioglu et al., 2018), sub-optimal alpha was observed for the SE sub-scale and was acceptable for the QC sub-scale, therefore cultural nuances may possibly influence individual sub-scale 
internal consistency against a backdrop of otherwise good psychometric properties and consistent factor structure. George and Mallery (2003) note that alpha levels of $>0.50$ are not unacceptable, however, in terms of future work with the S-BSS-R, further evaluation of the internal consistency properties of this QC sub-scale is desirable in the event future revision of sub-scale items is required.

The current investigation had a number of limitations. These include limits on the amount of socio-demographic data available, thus it was not possible to examine the influence of employment, marital or educational status on S-BSS-R scores. Additionally, all participants in the current study gave birth within the public health system, thus no information was available on the potential impact on birth satisfaction of private health service provision models. Extending the current research focus to address these inherent shortcomings is desirable and achievable through future research endeavours that use the S-BSS-R. 


\section{References}

Ali, E., 2018. Women’s experiences with postpartum anxiety disorders: A narrative literature review. Int. J. Womens. Health. 10, 237-249. https://doi.org/10.2147/IJWH.S158621

Barbosa-Leiker, C., Fleming, S., Hollins Martin, C.J., Martin, C.R., 2015. Psychometric properties of the Birth Satisfaction Scale-Revised (BSS-R) for US mothers. J. Reprod. Infant Psychol. 33, 504-511. https://doi.org/10.1080/02646838.2015.1024211

Bell, A.F., Andersson, E., 2016. The birth experience and women’s postnatal depression: A systematic review. Midwifery 39, 112-123. https://doi.org/10.1016/j.midw.2016.04.014

Bentler, P.M., 1990. Comparative fit indexes in structural models. Psychol. Bull. 107, 238-246. https://doi.org/10.1037/0033-2909.107.2.238

Blazquez, R.A., Ferrandiz, E.F., Moya, S.P., Caballero, V.G., Corchon, S., 2018. Translation, adaptation and psychometric validation of The Preterm Birth Experience and Satisfaction Scale (P-BESS) into Spanish. Midwifery 66, 148-154. https://doi.org/10.1016/j.midw.2018.08.007

Brown, T., 2015. Confirmatory Factor Analysis for Applied Research, 2nd ed. Guilford Press, New York.

Bryanton, J., Gagnon, A.J., Johnston, C., Hatem, M., 2008. Predictors of women’s perceptions of the childbirth experience. JOGNN - J. Obstet. Gynecol. Neonatal Nurs. 37, 24-34. https://doi.org/10.1111/j.1552-6909.2007.00203.x 
Burduli, E., Barbosa-Leiker, C., Fleming, S., Hollins Martin, C.J., Martin, C.R., 2017. Cross-cultural invariance of the Birth Satisfaction Scale-Revised (BSS-R): comparing UK and US samples. J. Reprod. Infant Psychol. 35 (3), 248-260. https://doi.org/10.1080/02646838.2017.1310374

Christiaens, W., Verhaeghe, M., Bracke, P., 2010. Pain acceptance and personal control in pain relief in two maternity care models: A cross-national comparison of Belgium and the Netherlands. BMC Health Serv. Res. 10, 268. https://doi.org/10.1186/1472-6963-10-268

Cohen, J., 1977. Statistical Power Analysis for the Behavioral Sciences, Rev. ed. Academic Press, New York.

Cohen, S., Kamarck, T., Mermelstein, R., 1983. A Global Measure of Perceived Stress. Journal of Health and Social Behavior. 24, 385-396.

Conde, A.A., Figueiredo, B., Costa, R., Pacheco, A., Pais, Á., 2008. Perception of the childbirth experience: Continuity and changes over the postpartum period. J. Reprod. Infant Psychol. 26, 139-154. https://doi.org/10.1080/02646830801918414

Cronbach, L.J., 1951. Coefficient alpha and the internal structure of tests. Psychometrika 16, 297-334. https://doi.org/10.1007/BF02310555

Dennis, C.L., Brown, H.K., Wanigaratne, S., Vigod, S.N., Grigoriadis, S., Fung, K., Marini, F., Brennenstuhl, S., 2017. Determinants of comorbid depression and anxiety postnatally: A longitudinal cohort study of Chinese-Canadian women. J. Affect. Disord. 227, 24-30. https://doi.org/10.1016/j.jad.2017.09.033

Diedenhofen, B., Musch, J., 2015. Cocor: A comprehensive solution for the statistical comparison of correlations. PLoS One 10. 
https://doi.org/10.1371/journal.pone.0121945

Dikmen-Yildiz, P., Ayers, S., Phillips, L., 2017. Depression, anxiety, PTSD and comorbidity in perinatal women in Turkey: A longitudinal population-based study. Midwifery 55, 29-37. https://doi.org/10.1016/j.midw.2017.09.001

Fleming, S.E., Donovan-Batson, C., Burduli, E., Barbosa-Leiker, C., Hollins Martin, C.J., Martin, C.R., 2016. Birth Satisfaction Scale/Birth Satisfaction ScaleRevised (BSS/BSS-R): A large scale United States planned home birth and birth centre survey. Midwifery 41, 9-15. https://doi.org/10.1016/j.midw.2016.07.008

Ford, E., Ayers, S., 2009. Stressful events and support during birth: The effect on anxiety, mood and perceived control. J. Anxiety Disord. 23, 260-268. https://doi.org/10.1016/j.janxdis.2008.07.009

Fowler, G., Patterson, D., 2013. Use of maternity surveys in improving the care experience -- a review of the evidence. Br. J. Midwifery 21, 410-415. https://doi.org/10.12968/bjom.2013.21.6.410

George, D., Mallery, P., 2003. SPSS for Windows Step by Step: A Simple Guide and Reference. 11.0 update, 4th ed. Allyn and Bacon, Boston, MA.

Goncu Serhatlioglu, S., Karahan, N., Hollins Martin, C.J., Martin, C.R., 2018. Construct and content validity of the Turkish Birth Satisfaction Scale - Revised (T-BSSR). J. Reprod. Infant Psychol. 36 (3), 235-245. https://doi.org/10.1080/02646838.2018.1443322

Goodman, P., Mackey, M.C., Tavakoli, A.S., 2004. Factors related to childbirth satisfaction. J. Adv. Nurs. 46, 212-219. https://doi.org/10.1111/j.13652648.2003.02981.x 
Goutaudier, N., Bertoli, C., Séjourné, N., Chabrol, H., 2018. Childbirth as a forthcoming traumatic event: pretraumatic stress disorder during pregnancy and its psychological correlates. J. Reprod. Infant Psychol. 1-12. https://doi.org/10.1080/02646838.2018.1504284

Guszkowska, M., 2014. The effect of exercise and childbirth classes on fear of childbirth and locus of labor pain control. Anxiety, Stress Coping 27, 176-189. https://doi.org/10.1080/10615806.2013.830107

Hollins Martin, C., Fleming, V., 2011. The birth satisfaction scale. Int. J. Health Care Qual. Assur. 24, 124-135. https://doi.org/10.1108/09526861111105086

Hollins Martin, C.J., Martin, C.R., 2014. Development and psychometric properties of the Birth Satisfaction Scale-Revised (BSS-R). Midwifery 30, 610-619. https://doi.org/10.1016/j.midw.2013.10.006

Hu, L.T., Bentler, P.M., 1999. Cutoff criteria for fit indexes in covariance structure analysis: Conventional criteria versus new alternatives. Struct. Equ. Model. 6, 155. https://doi.org/10.1080/10705519909540118

Jefford, E., Hollins Martin, C.J., Martin, C.R., 2018. Development and validation of the Australian version of the Birth Satisfaction Scale-Revised (BSS-R). J. Reprod. Infant Psychol. 36, 42-58. https://doi.org/10.1080/02646838.2017.1396302

Kline, P., 2000. A Psychometrics Primer. Free Association Books, London.

Kline, R.B., 2011. Principles and Practice of Structural Equation Modeling, 3rd ed. Guilford Press, London.

Konerding, U., 2016. Which kind of psychometrics is adequate for patient satisfaction questionnaires? Patient Prefer. Adherence 10, 2083-2090. 
https://doi.org/10.2147/PPA.S112398

Marín-Morales, D., Carmona-Monge, F.J., Peñacoba-Puente, C., Olmos Albacete, R., Toro Molina, S., 2013. Factor structure, validity, and reliability of the spanish version of the women's views of birth labour satisfaction questionnaire. Midwifery 29, 1339-1345. https://doi.org/10.1016/j.midw.2012.12.015

Martin, C.R., Hollins Martin, C.J., 2018. Sensitivity of the Birth Satisfaction ScaleRevised (BSS-R) to delivery type: A meta-analysis, Society for Reproductive and Infant Psychology Annual Conference, Lodz, Poland.

Martin, C.R., Hollins Martin, C.J., Burduli, E., Barbosa-Leiker, C., Donovan-Batson, C., Fleming, S.E., 2017. Measurement and structural invariance of the US version of the Birth Satisfaction Scale-Revised (BSS-R) in a large sample. Women and Birth 30, e172-e178. https://doi.org/10.1016/j.wombi.2016.11.006

Martin, C.R., Vardavaki, Z., Hollins Martin, C.J., 2016. Measurement equivalence of the Birth Satisfaction Scale-Revised (BSS-R): further evidence of construct validity. J. Reprod. Infant Psychol. 34, 394-402. https://doi.org/10.1080/02646838.2016.1184747

Mpembeni, R.N.M., Killewo, J.Z., Leshabari, M.T., Massawe, S.N., Jahn, A., Mushi, D., Mwakipa, H., 2007. Use pattern of maternal health services and determinants of skilled care during delivery in Southern Tanzania: Implications for achievement of MDG-5 targets. BMC Pregnancy Childbirth 7. https://doi.org/10.1186/14712393-7-29

Pampaka, D., Papatheodorou, S.I., AlSeaidan, M., Al Wotayan, R., Wright, R.J., Buring, J.E., Dockery, D.W., Christophi, C.A., 2018. Postnatal depressive symptoms in women with and without antenatal depressive symptoms: results from 
a prospective cohort study. Arch. Womens. Ment. Health 1-11.

https://doi.org/10.1007/s00737-018-0880-8

Remor, E., 2006. Psychometric Properties of a European Spanish Version of the Perceived Stress Scale (PSS). Span. J. Psychol. 9, 86-93. https://doi.org/10.1017/S1138741600006004

Shlomi Polachek, I., Dulitzky, M., Margolis-Dorfman, L., Simchen, M.J., 2016. A simple model for prediction postpartum PTSD in high-risk pregnancies. Arch. Womens. Ment. Health 19, 483-490. https://doi.org/10.1007/s00737-015-0582-4

Srivastava, A., Avan, B.I., Rajbangshi, P., Bhattacharyya, S., 2015. Determinants of women’s satisfaction with maternal health care: A review of literature from developing countries. BMC Pregnancy Childbirth 15, 1-12. https://doi.org/10.1186/s12884-015-0525-0

Steiger, J.H., Lind, J., 1980. Statistically-based tests for the number of common factors, Annual Spring Meeting of the Psychometric Society, Iowa City, USA.

Striebich, S., Mattern, E., Ayerle, G.M., 2018. Support for pregnant women identified with fear of childbirth (FOC)/tokophobia - A systematic review of approaches and interventions. Midwifery 61, 97-115. https://doi.org/10.1016/j.midw.2018.02.013

The International Consortium for Health Outcome Measurement, 2016. Pregnancy and Childbirth Standard Set and Reference Guide.

Van der Gucht, N., Lewis, K., 2015. Women’s experiences of coping with pain during childbirth: A critical review of qualitative research. Midwifery 31, 349-358. https://doi.org/10.1016/j.midw.2014.12.005

Vardavaki, Z., Hollins Martin, C.J., Martin, C.R., 2015. Construct and content validity 
of the Greek version of the Birth Satisfaction Scale (G-BSS). J. Reprod. Infant Psychol. 33, 488-503. https://doi.org/10.1080/02646838.2015.1035235

Whitburn, L.Y., Jones, L.E., Davey, M.A., Small, R., 2014. Women’s experiences of labour pain and the role of the mind: An exploratory study. Midwifery 30, 10291035. https://doi.org/10.1016/j.midw.2014.04.005 
Table 1. Mean, standard deviation and distributional characteristics of S-BSS-R sub-scales and total score and comparison with mean (Mu) UK BSS-R scores reported by Hollins Martin and Martin (2014) using one-sample $t$-test ( $\mathrm{df}=201)$. se = standard error of kurtosis, CI = Confidence Interval.

\begin{tabular}{lccccccccccc}
\hline Subscale & Mean & SD & Min Max & Skew & Kurtosis & se & Mu & $t$ & $p$ & $95 \%$ CI \\
\hline Stress & 9.36 & 3.68 & 0 & 16 & -0.39 & -0.41 & 0.26 & 9.70 & 1.83 & 0.19 & $8.85-9.87$ \\
Attributes & 4.76 & 2.01 & 0 & 8 & -0.32 & -0.44 & 0.14 & 4.90 & 1.01 & 0.32 & $4.48-5.04$ \\
Quality & 13.94 & 2.01 & 7 & 16 & -0.80 & 0.16 & 0.14 & 13.76 & 1.28 & 0.20 & $13.66-14.22$ \\
Total Score & 28.05 & 6.22 & 12 & 40 & -0.30 & -0.42 & 0.44 & 28.36 & 0.70 & 0.49 & $27.19-28.92$ \\
\hline
\end{tabular}


Table 2. Mean, standard deviation and distributional characteristics of individual S-BSS-R items. se = standard error of kurtosis.

\begin{tabular}{|c|c|c|c|c|c|c|c|c|c|}
\hline Item & Item content & Domain* & Mean & SD & Min & Max & Skew & Kurtosis & se \\
\hline \multirow[t]{2}{*}{ BSS-R 3} & The delivery room staff encouraged me to make & QC & 2.99 & 1.10 & 0 & 4 & -1.12 & 0.64 & 0.08 \\
\hline & decisions about how I wanted my birth to progress & & & & & & & & \\
\hline \multirow[t]{2}{*}{ BSS-R 5} & I felt well supported by staff during my labour and & QC & 3.68 & 0.55 & 1 & 4 & -1.68 & 2.86 & 0.04 \\
\hline & birth & & & & & & & & \\
\hline BSS-R 6 & The staff communicated well with me during labour & QC & 3.69 & 0.59 & 0 & 4 & -2.44 & 8.72 & 0.04 \\
\hline BSS-R 9 & I was not distressed at all during labour & $\mathrm{SE}$ & 2.19 & 1.19 & 0 & 4 & 0.04 & -1.09 & 0.08 \\
\hline BSS-R 10 & The delivery room was clean and hygienic & QC & 3.58 & 0.71 & 0 & 4 & -2.05 & 4.91 & 0.05 \\
\hline
\end{tabular}

*Domain of the S-BSS-R. SE = Stress experienced during child-bearing, WA = Women's attributes, QC = Quality of Care. 
Table 3. Correlations of S-BSS-R sub-scales and total score and comparison with original UK BSS-R (Hollins Martin and Martin, 2014).

\begin{tabular}{lccccc}
\hline Scale combination & Spanish $r$ & UK $r$ & Z & $95 \%$ CI & $p$ \\
\hline Stress-Attributes & 0.61 & 0.57 & 0.63 & $(-0.09-0.16)$ & 0.53 \\
Stress-Quality & 0.39 & 0.26 & 1.50 & $(-0.04-0.30)$ & 0.13 \\
Attributes-Quality & 0.29 & 0.35 & 0.69 & $(-0.23-0.11)$ & 0.49 \\
Total score-Stress & 0.91 & 0.86 & 2.41 & $(0.01-0.09)$ & $0.02^{*}$ \\
Total score-Attributes & 0.78 & 0.80 & 0.60 & $(-0.09-0.05)$ & 0.55 \\
Totals score-Quality & 0.64 & 0.63 & 0.19 & $(-0.09-0.12)$ & 0.85 \\
\hline
\end{tabular}

${ }^{*} p<0.05$. 
Table 4. Comparison of S-BSS-R total and sub-scale scores as a function of birth delivery type. Standard deviations are in parentheses, degrees of freedom $=200, \mathrm{CI}=$ confidence interval. Intervention group comprised, planned Ceasarean section $\mathrm{N}=12$, emergency Ceasarean section $\mathrm{N}=19$, forceps $\mathrm{N}=5$, vacuum cup $\mathrm{N}=29$, spatula $\mathrm{N}=3$, and breech birth $\mathrm{N}=1$.

\begin{tabular}{lccccccccc}
\hline BSS-R Scale & $\begin{array}{c}\text { Unassisted } \\
\text { vaginal } \\
\text { delivery } \\
(\mathrm{N}=133)\end{array}$ & $\begin{array}{c}\text { Intervention } \\
\text { delivery }\end{array}$ & 95\% CI & $t$ & $p$ & Hedges g & Hedges g 95\% CI & Effect size* \\
\hline Stress & $10.60(2.99)$ & $6.96(3.70)$ & $2.69-4.60$ & 7.56 & $<0.001$ & 1.12 & $0.81-1.43$ & Large \\
Attributes & $5.08(2.06)$ & $4.15(1.78)$ & $0.35-1.51$ & 3.18 & 0.002 & 0.47 & $0.17-0.77$ & Small \\
Quality & $14.29(1.83)$ & $13.28(2.17)$ & $0.44-1.58$ & $3.49<0.001$ & 0.52 & $0.22-0.81$ & Medium \\
Total score & $29.96(5.38)$ & $24.38(6.10)$ & $3.94-7.24$ & $6.68<0.001$ & 0.99 & $0.68-1.30$ & Large \\
\hline
\end{tabular}

* Note: Effect size conventions, 0.2 = small, 0.5 = medium, 0.8 = large (Cohen, 1977). 
Table 5. Comparison of S-BSS-R total and sub-scale scores as a function of pain control received. Standard deviations are in parentheses, degrees of freedom $=197, \mathrm{CI}=$ confidence interval. Pain control group comprised, epidural N=139, nitrous oxide $\mathrm{N}=13$, massage $\mathrm{N}=6$. Note: $\mathrm{N}=3$ missing cases due to incomplete pain control data.

\begin{tabular}{llccccccc}
\hline BSS-R Scale & $\begin{array}{l}\text { No pain } \\
\text { control } \\
(\mathrm{N}=41)\end{array}$ & $\begin{array}{c}\text { Pain } \\
\text { control } \\
(\mathrm{N}=158)\end{array}$ & $95 \% \mathrm{CI}$ & $t$ & $p$ & Hedges g & Hedges g 95\% CI & Effect size \\
\hline Stress & $11.51(2.84)$ & $8.79(3.69)$ & $1.51-3.95$ & $4.40<0.001$ & 0.77 & $0.41-1.12$ & Medium \\
Attributes & $5.42(2.04)$ & $4.62(1.98)$ & $0.11-1.48$ & 2.27 & 0.02 & 0.40 & $0.04-0.75$ & Small \\
Quality & $13.95(2.21)$ & $13.94(1.96)$ & $-0.69-0.71$ & 0.02 & 0.98 & 0.004 & $-0.34-0.35$ & Negligible \\
Total score & $30.88(5.82)$ & $27.35(6.18)$ & $1.42-5.64$ & 3.30 & 0.001 & 0.58 & $0.23-0.93$ & Medium \\
\hline
\end{tabular}


Table 6. Cronbach’s alpha of S-BSS-R sub-scales and total score and comparison

with original UK BSS-R (Hollins Martin and Martin, 2014). Degrees of freedom = 1 .

\begin{tabular}{lcccc}
\hline Subscale & Spanish alpha & UK alpha & $\chi^{2}$ & $p$ \\
\hline Stress & 0.70 & 0.71 & 0.04 & 0.85 \\
Attributes & 0.57 & 0.64 & 0.56 & 0.46 \\
Quality & 0.55 & 0.74 & 9.57 & $0.002^{*}$ \\
Total score & 0.77 & 0.79 & 0.36 & 0.55 \\
\hline
\end{tabular}

${ }^{*} p<0.005$. 